\author{
A) Check for updates \\ Cite this: Nanoscale, 2020, 12, 16730
}

\section{Nitric oxide-dependent biodegradation of graphene oxide reduces inflammation in the gastrointestinal tract $\uparrow$}

\author{
Received 11th May 2020, \\ Accepted 7th August 2020 \\ DOI: $10.1039 /$ dOnr03675g \\ rsc.li/nanoscale
}

\author{
Guotao Peng, (D) a Marcelo F. Montenegro, (DD ${ }^{\mathrm{b}}$ Chifundo N. M. Ntola, ${ }^{\mathrm{c}}$ \\ Sandra Vranic, ${ }^{c}$ Kostas Kostarelos, (D) c,d Carmen Vogt, ${ }^{e}$ Muhammet S. Toprak, (DD e \\ Tianbo Duan, ${ }^{f}$ Klaus Leifer, (D ${ }^{f}$ Lars Bräutigam, ${ }^{g}$ Jon O. Lundberg ${ }^{b}$ and \\ Bengt Fadeel $\mathbb{D}$ *a
}

\begin{abstract}
Understanding the biological fate of graphene-based materials such as graphene oxide (GO) is crucial to assess adverse effects following intentional or inadvertent exposure. Here we provide first evidence of biodegradation of GO in the gastrointestinal tract using zebrafish as a model. Raman mapping was deployed to assess biodegradation. The degradation was blocked upon knockdown of nos2a encoding the inducible nitric oxide synthase (iNOS) or by pharmacological inhibition of NOS using L-NAME, demonstrating that the process was nitric oxide (NO)-dependent. NOdependent degradation of GO was further confirmed in vitro by combining a superoxide-generating system, xanthine/xanthine oxidase (X/XO), with an NO donor (PAPA NONOate), or by simultaneously producing superoxide and NO by decomposition of SIN-1. Finally, by using the transgenic strain $\mathrm{Tg}$ (mpx:eGFP) to visualize the movement of neutrophils, we could show that inhibition of the degradation of GO resulted in increased neutrophil infiltration into the gastrointestinal tract, indicative of inflammation.
\end{abstract}

Graphene and its derivatives have been intensively explored for a multitude of biomedical and other applications. This, therefore, mandates a comprehensive assessment of the potential impact of these materials on human health. ${ }^{1}$ In recent years, attention has been focused on the biological fate of graphenebased materials, including their potential biodegradability. Thus, enzymatic degradation of graphene oxide (GO) by plant

\footnotetext{
${ }^{a}$ Institute of Environmental Medicine, Karolinska Institutet, Stockholm, Sweden. E-mail: bengt.fadeel@ki.se

${ }^{b}$ Department of Physiology and Pharmacology, Karolinska Institutet, Stockholm, Sweden

${ }^{c}$ National Graphene Institute, and Faculty of Biology, Medicine and Health, University of Manchester, Manchester, UK

${ }^{d}$ Catalan Institute of Nanoscience and Nanotechnology (ICN2), Barcelona, Spain

${ }^{e}$ Department of Applied Physics, KTH Royal Institute of Technology, Stockholm, Sweden

${ }^{f}$ Department of Engineering Sciences, Uppsala University, Uppsala, Sweden

${ }^{g}$ Comparative Medicine, Karolinska Institutet, Stockholm, Sweden

$\dagger$ Electronic supplementary information (ESI) available. See DOI: 10.1039/ d0nr03675g
}

and animal peroxidases, including myeloperoxidase (MPO), has been documented. ${ }^{2,3}$ Purified human MPO was shown to degrade highly dispersed GO, but failed to metabolize aggregated GO. ${ }^{3}$ Furthermore, we demonstrated in a previous study that primary human neutrophils are capable of degrading GO when activated to undergo degranulation with release of $\mathrm{MPO},{ }^{4}$ while GO functionalized with a chemotactic peptide triggered neutrophil activation with subsequent degradation of GO. ${ }^{5}$ Single- and few-layer graphene is also susceptible to enzymatic degradation though the process was found to be less efficient as compared to GO. ${ }^{6}$

Previous studies on single- and multi-walled carbon nanotubes (CNTs) have revealed the propensity of these materials to undergo peroxidase-mediated biodegradation. ${ }^{7-10}$ Furthermore, CNTs may also undergo peroxynitrite $\left(\mathrm{ONOO}^{-}\right)$dependent biodegradation in macrophages ${ }^{11,12}$ and chondrocytes. $^{13}$ Peroxynitrite can be generated by the rapid reaction of superoxide radicals with nitric oxide (NO) according to the following reaction:

$$
\mathrm{O}_{2}^{\cdot-}+\mathrm{NO}^{\cdot} \rightarrow \mathrm{ONOO}^{-}
$$

The peroxynitrite anion produced in this manner displays high diffusibility, ${ }^{14}$ obviating the need for a direct interaction of CNTs with the participating enzymes. ${ }^{11}$ However, no study has been conducted to investigate the possibility of NO-driven biodegradation of GO. Here we studied GO produced by the Hummers' method to investigate the in vitro and in vivo degradability. The physicochemical characterization results are summarized in Table S1.† Based on the evaluation of GO by TEM, SEM, optical microscopy and AFM, we found that $90 \%$ of the sheets ranged between 0.1 to $15 \mu \mathrm{m}$ in lateral dimensions (representative TEM images are shown in Fig. 1a). AFM analysis revealed that the thickness of the GO sheets was between 1 to $2 \mathrm{~nm}$, allowing us to conclude that GO was mainly present as single- or few-layer sheets in water and after incubation for $24 \mathrm{~h}$ in E3 medium (Fig. 1b and c). Note that the spikes in thickness following incubation in E3 medium are due to 
a

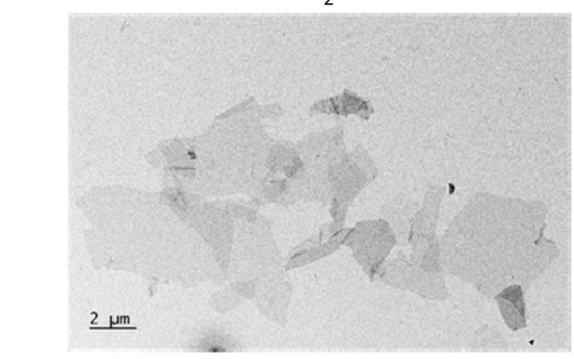

b

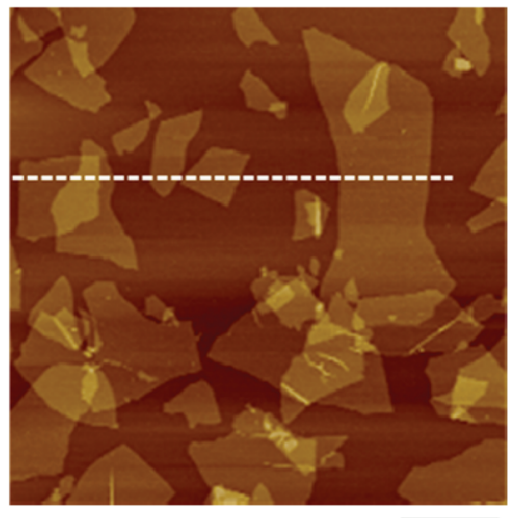

$\overline{1.0 \mu \mathrm{m}}$

c

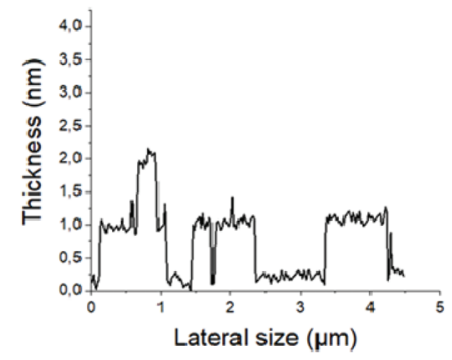

E3 medium
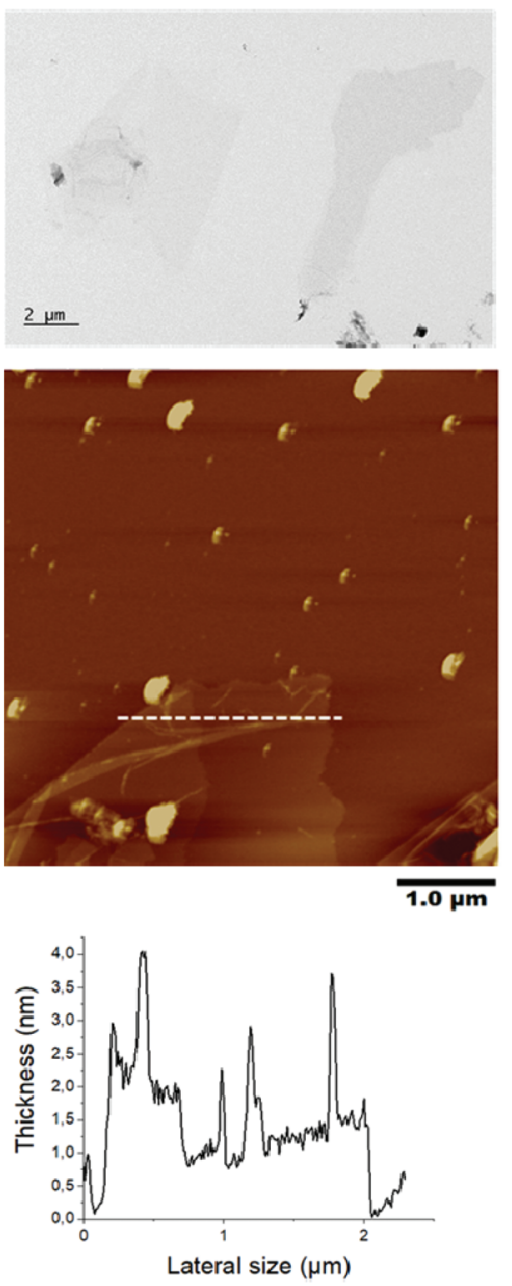

Fig. 1 Structural characterization of GO sheets after incubation in E3 medium versus water using transmission electron microscopy (TEM) (a) and atomic force microscopy (AFM) (b) height image and (c) cross section analysis highlighted by the line in the height image.

wrinkling and folding of GO sheets as a result of incubation in a high salt containing medium.

Inhalation is the main route of unintentional exposure to engineered nanomaterials, and the lungs therefore represent the most immediate target organ for their toxic effects. However, extrapulmonary effects of inhaled nanomaterials including effects on the gastrointestinal (GI) tract have been reported; additionally, nanomaterials can be ingested leading to a direct exposure of the GI tract. ${ }^{15}$ Despite the potential implications on human health, the impact of nanomaterials on the GI tract has been largely overlooked. ${ }^{16}$ We focused here on oral exposure to GO. It is well known that NO is produced in the GI tract through enzymatic and non-enzymatic reactions, ${ }^{17}$ and we hypothesized that GO may undergo NO-dependent biodegradation (Fig. 2a). To address this question, we used zebrafish embryos that have emerged in recent years as a robust in vivo model in toxicology and drug discovery. ${ }^{18}$ The degradation of GO in zebrafish larvae ( 5 days postfertilization, dpf) was evaluated using confocal Raman mapping. To investigate the mechanism of biodegradation of GO in the GI tract, we employed two approaches: (i) downregulation of the inducible NO synthase (iNOS) by using morpholino oligonucleotides directed against nos $2 a$; and (ii) inhibition of NOS by using the cell-permeable inhibitor, $N$-omega-nitro-L-arginine methyl ester hydrochloride (L-NAME). The successful downregulation of nos $2 a$ was documented by RT-qPCR (Fig. 2b). As demonstrated in Fig. 2c, GO accumulated in the GI tract and could be detected on the basis of its characteristic Raman signature, i.e., the $\mathrm{D}$ band $\left(1354 \mathrm{~cm}^{-1}\right), \mathrm{G}$ band $\left(1582 \mathrm{~cm}^{-1}\right)$, and $2 \mathrm{D}$ band $\left(2690 \mathrm{~cm}^{-1}\right) .{ }^{19}$ The background signal of the fish tissues did not interfere with the detection of GO by Raman (data not shown). Importantly, our interventions (shown schematically in Fig. 2a) resulted in a pronounced change in the intensity of the D and $G$ band when compared to untreated, GO-exposed fish, indicating that GO was biodegraded in the latter case through an NOdependent mechanism (Fig. 2d). It is worth noting that a previous study showed no degradation of GO when immersed in simulated digestive juices representative of the GI tract, as evaluated by Raman analysis. ${ }^{20}$ This allows us to rule out a role of gastrointestinal fluids in the degradation of GO. 
a

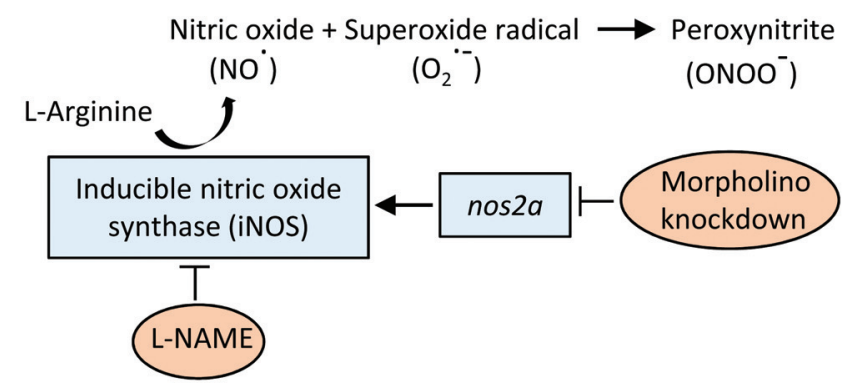

C

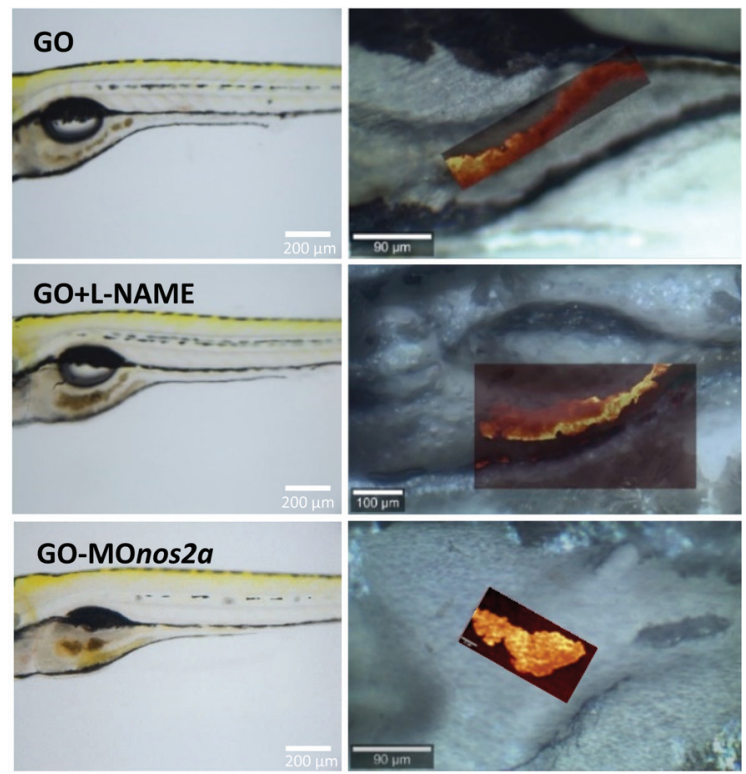

b

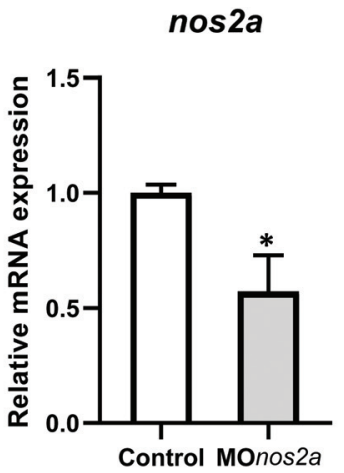

d

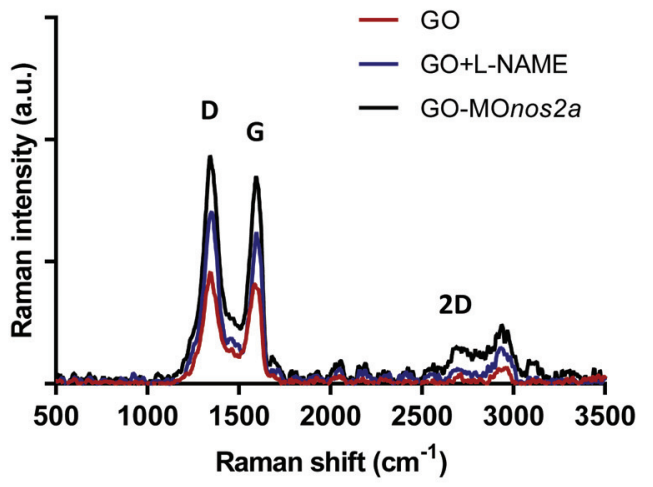

Fig. 2 NO-dependent biodegradation of GO in vivo. (a) Schematic diagram showing the generation of nitric oxide (NO) by iNOS followed by the reaction of superoxide radicals and NO to form the potent oxidant, peroxynitrite. (b) Relative mRNA expression level of nos2a following knockdown of nos2a. (c) Raman confocal mapping of GO in zebrafish. GO was found to accumulate in the gut upon oral exposure, as demonstrated by optical and Raman confocal images. (d) Zebrafish larvae at $5 \mathrm{dpf}$ were exposed to $\mathrm{GO}$ at $5 \mu \mathrm{g} \mathrm{mL} \mathrm{m}^{-1}$ for $24 \mathrm{~h}$. Morpholino oligonucleotides (MO) were injected into one-cell stage zebrafish embryos to downregulate nos2a expression. L-NAME was used to inhibit NOS in conventional fish. Each spectrum represents the average of 10.000 spectra across the whole area scan.

To support the role of NO in the biodegradation process, we measured NO production in vivo by using the fluorescent probe, DAF-FM-DA. Bacterial lipopolysaccharide (LPS) was used to trigger NO production (Fig. 3a). NO production was suppressed by L-NAME (Fig. 3b). Exposure to GO also led to an increase in NO (Fig. 3b). Importantly, knockdown of nos $2 a$ with morpholino oligos resulted in a decline in LPS- and GOinduced NO production (Fig. 3b; representative images of zebrafish embryos are shown in panel a). We also detected peroxynitrite generation in vivo by using DAX-J2 PON Green, a fluorescent probe with high selectivity for peroxynitrite and negligible reactivity with other relevant species including NO. Peroxynitrite production was observed in vivo in response to GO and to a lesser extent with LPS and the signal was suppressed by L-NAME (Fig. S1†).

To further confirm the degradation of GO and provide more insight regarding the NO-dependent reaction, we performed in vitro experiments by using two peroxynitrite-generating systems, i.e., simultaneous production of superoxide radicals and NO by decomposition of 3-morpholinosydnonimine hydrochloride (SIN-1), or the combination of a superoxide-generating system, xanthine/xanthine oxidase (X/XO), with an NO donor (PAPA NONOate). ${ }^{11}$ As shown in Fig. 4a, GO degradation after 5 days of continuous incubation in the presence of SIN-1 or PAPA NONOate-X/XO was visually detectable as the suspensions turned translucent in both cases. To more objectively document the degradation, we performed Raman spectroscopy. The characteristic D and G bands of GO were remarkably diminished in both systems suggesting a near-complete loss of structure of GO (Fig. 4b). The topography and structure of GO incubated with the two peroxynitrite-generating systems was further characterized by TEM (Fig. 4c) and AFM (Fig. 4d). Specifically, after PAPA NONOate-X/XO treatment, the typical morphology of the GO sheets disappeared, and only small frag- 

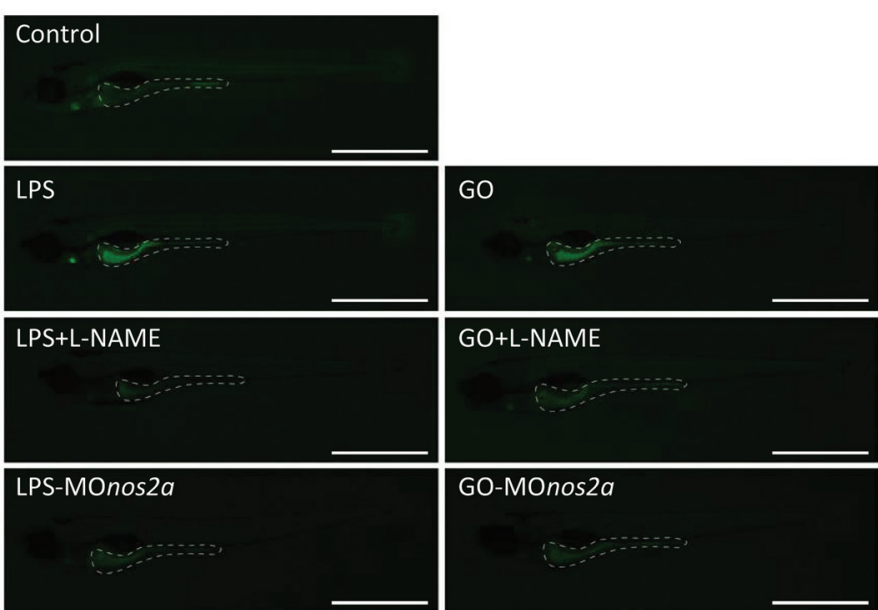

b

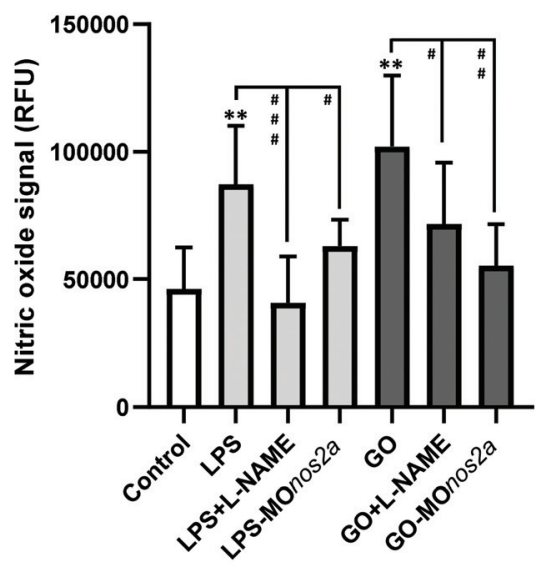

Fig. 3 NO production in zebrafish. (a) Representative fluorescence images of NO production in zebrafish gut, represented as the corrected gut fluorescence determined by DAF-FM-DA assay. The gut is indicated by the white dashed line. (b) Quantification of NO production determined by DAF-FM-DA assay. NO production was induced by LPS and GO, while L-NAME and downregulation of nos2a significantly inhibited NO production. Student's $t$-test was used to evaluate the statistical significance between treated fish and untreated controls $\left({ }^{*} p<0.05, * * p<0.01, * * * p<0.001\right)$, as well as the LPS and GO-exposed fish with/without NOS inhibition/suppression (\# $p<0.05, \# \# p<0.01, \# \# \# p<0.001$ ).

a

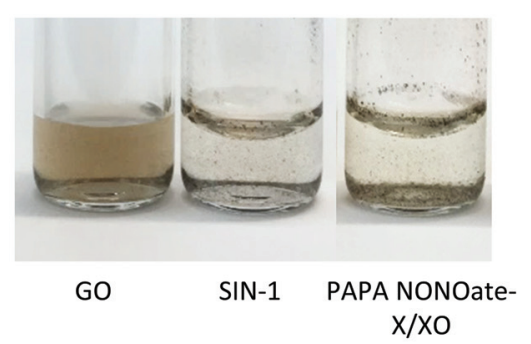

C
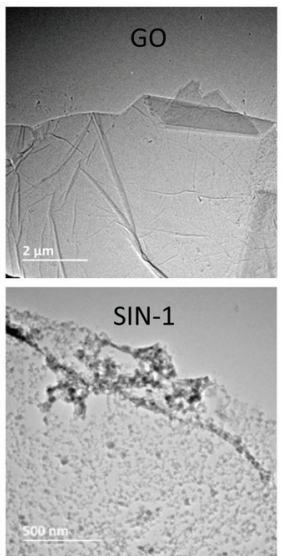

b

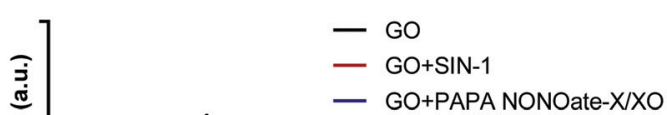

d

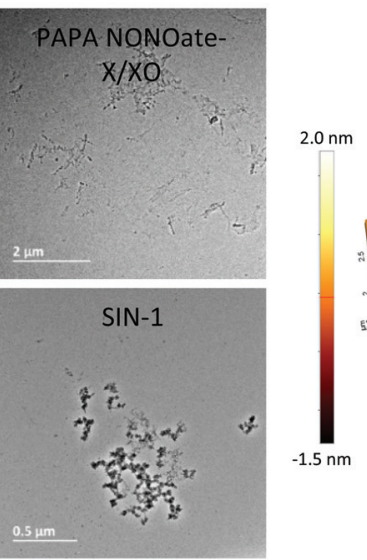

- GO+SIN-1

- GO+PAPA NONOate-X/XO

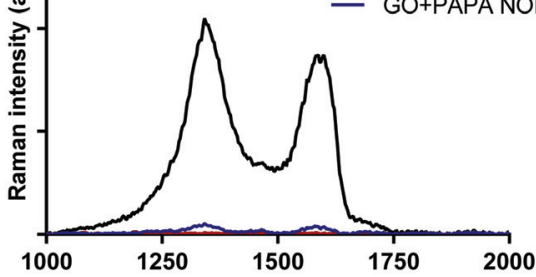

Raman shift $\left(\mathrm{cm}^{-1}\right)$
GO

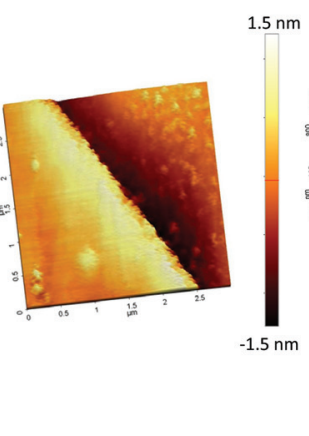

SIN-1

PAPA NONOate-X/XO

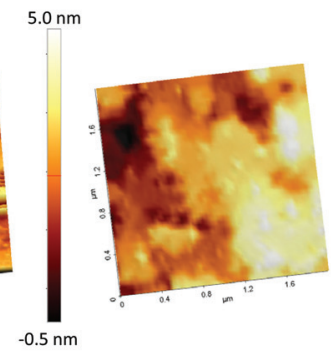

Fig. 4 NO-dependent degradation of GO in vitro. (a) Visual evidence of degradation upon incubation of GO with the peroxynitrite donor, SIN-1 or PAPA NONOate-X/XO for 5 days. (b) Raman analysis of GO after incubation with SIN-1 and PAPA NONOate-X/XO for 5 days showed almost complete degradation of GO as evidenced by loss of the characteristic D and G bands. (c) Representative TEM images of untreated GO versus GO exposed to SIN-1 and PAPA NONOate-X/XO for 5 days. Numerous defects in the GO sheets are visible in SIN-1 exposed samples, in particular, as well as in GO exposed to PAPA NONOate-X/XO. (d) AFM height contrast images of representative GO samples with or without SIN-1 and PAPA NONOate-X/XO. The surface of pristine GO is flat, with a mean square roughness (MSR) of $0.112 \mathrm{~nm}$ and a thickness of $1-2 \mathrm{~nm}$, while in GO sheets exposed to SIN-1, several defects of lateral sizes ranging from $50 \mathrm{~nm}$ to $200 \mathrm{~nm}$ appear, which could be attributed to the degradation of GO. Furthermore, GO incubated with PAPA NONOate-X/XO shows greater roughness (MSR $=1.655 \mathrm{~nm}$ ) and an increase of the thickness of GO, which could be due to the aggregation of the degraded smaller flakes of GO. 
ments could be observed. In addition, SIN-1 treatment led to numerous perforations of the GO sheets, as shown by TEM, and these defects were also seen by AFM. To confirm that the two systems were functional, we monitored NO and peroxynitrite production based on real-time chemiluminescence and fluorescence detection, respectively. PAPA NONOate induced a robust and stable $\mathrm{NO}$ signal, and the addition of $\mathrm{X} / \mathrm{XO}$ was able to decrease the NO signal, likely due to the rapid reaction between NO and the superoxide radicals (Fig. 5a). Additionally, continuous treatment of GO revealed that PAPA NONOate alone was able to decrease the optical density of the GO suspensions, indicative of GO degradation, while $\mathrm{X} / \mathrm{XO}$ alone did not exert observable effects (Fig. S2a †). Raman analysis confirmed these results (Fig. S2b $\dagger$ ). On the other hand, the peroxynitrite donor SIN-1 did not generate a NO signal (at $300 \mu \mathrm{M}$ ), though there was a slight increase when a higher dose $(900 \mu \mathrm{M})$ was applied (Fig. 5b). Instead, the fluorescence intensity of DAX-J2 PON Green increased in a time- and dose-dependent manner upon reacting with SIN-1, clearly showing that peroxynitrite was formed, while PAPA NONOate (an NO donor) generated a negligible signal (Fig. 5c). Collectively, these results suggest that the degradation of GO by PAPA NONOate$\mathrm{X} / \mathrm{XO}$ may be attributed to NO, but not to superoxide radicals, and that the decomposition of SIN-1 produces superoxide and $\mathrm{NO}$ resulting in rapid formation of $\mathrm{ONOO}^{-}$, which 'digests' GO. One may ask what the potential advantages or disadvantages would be of NO-driven degradation of GO. One important aspect is that NO-driven degradation does not require direct interaction with the participating enzymes which means that cellular uptake of the nanomaterial is not required. Instead, NO released from gastrointestinal cells may diffuse to the gut lumen and 'attack' GO, leading to its degradation. On the other hand, a possible limitation is that the kinetics of NO-driven degradation are evidently slower than those of MPO. ${ }^{4}$

Only a few attempts have been made to assess the biological consequences of nanomaterial degradation or the lack thereof. Kagan et al. ${ }^{21}$ found that ex vivo degraded CNTs induced less pulmonary inflammation when aspirated into the lungs of mice whereas non-degraded CNTs triggered granuloma for- a

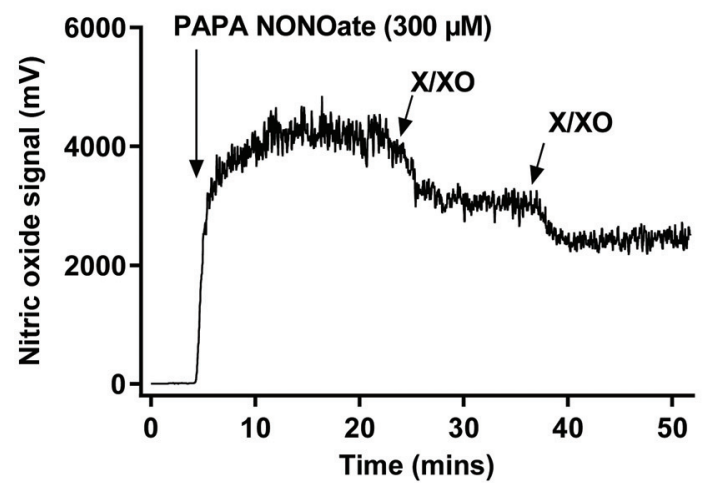

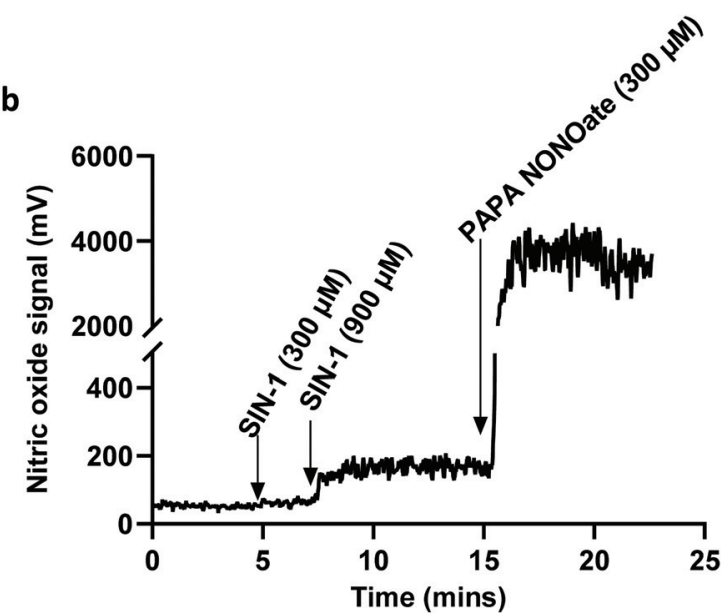

Time (mins)

C

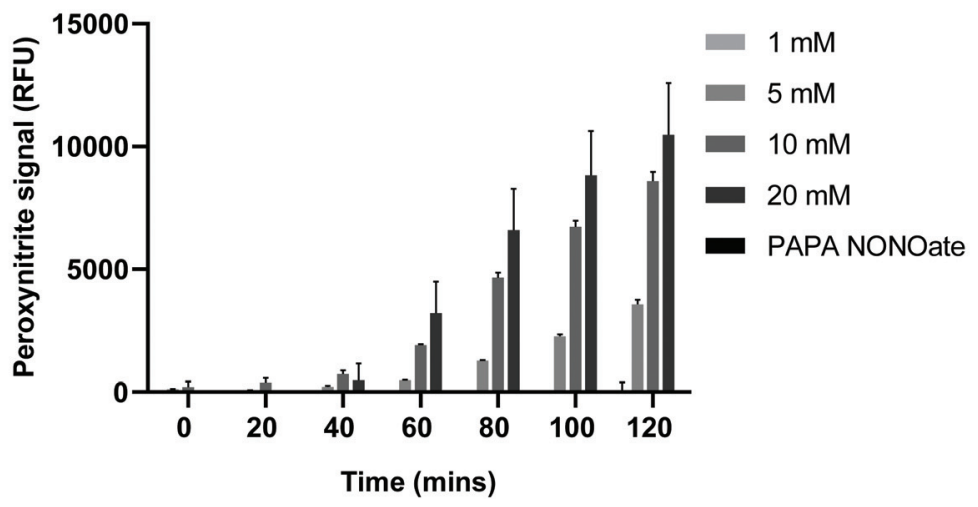

Fig. 5 Detection of NO and peroxynitrite using in vitro systems. (a) The NO donor, 1-propamine 3-(2-hydroxy-2-nitroso-1-propylhydrazine) (PAPA) NONOate, elicited a significant NO signal and the introduction of the superoxide-generating system xanthine/xanthine oxidase (X/XO) resulted in a decrease of the signal, indicative of peroxynitrite generation. (b) SIN-1 triggered a very low NO signal when compared with PAPA NONOate. (c) SIN-1 generated peroxynitrite in a dose- and time-dependent manner, while no peroxynitrite was observed when adding the NO donor PAPA NONOate (at $10 \mathrm{mM}$ ). 
mation. In a subsequent study, inflammatory and fibrotic responses to CNTs were aggravated in MPO-deficient mice when compared to wild-type mice. ${ }^{22}$ We recently reported that MPO-degraded GO was non-cytotoxic and did not exert DNA damage in cultured human lung cells, ${ }^{4}$ but in vivo responses were not evaluated in the latter study. Here we asked whether biodegradation would alter inflammatory responses induced by GO in the GI tract. To address this, we employed transgenic zebrafish, $T g$ (mpx:eGFP), expressing fluorescent GFP under a neutrophil-specific promoter to visualize neutrophil infiltration, ${ }^{23}$ an acknowledged marker of inflammation. As illustrated in Fig. 6a, the GFP-positive neutrophils were mostly located in the blood vessels in control fish. Following GO exposure, neutrophils were found to infiltrate the GI tract (Fig. 6a). Interestingly, in zebrafish exposed to GO in the presence of L-NAME, significantly more neutrophils migrated towards the GI tract as compared to fish exposed to GO alone (Fig. 6b and c). However, L-NAME alone had no impact on neutrophil migration (Fig. 6c). These results suggest that NO- dependent degradation of GO which is suppressed by L-NAME serves to reduce the inflammatory response triggered by ingested GO. We found that the recruited neutrophils were mainly located in the lamina propria (beneath the epithelium) while very few GFP-positive cells infiltrated into the gut lumen (Fig. S3†). Therefore, direct interactions between neutrophils and GO seem unlikely.

The fact that the major organ systems are conserved in zebrafish $^{18}$ provides an opportunity to decipher potential health effects of nanomaterials in humans. Specifically, zebrafish may serve as an alternative to mammalian models for the study of inflammation, as the cellular and humoral machinery that regulates immune responses is conserved. In the early stages of development, zebrafish rely on the innate immune system to protect against infections or injuries. The present study shows that the presence of GO is 'sensed' in the gut leading zebrafish larvae to mount a response to the offending agent. However, the implications of the present findings for other species including humans can only be speculated upon

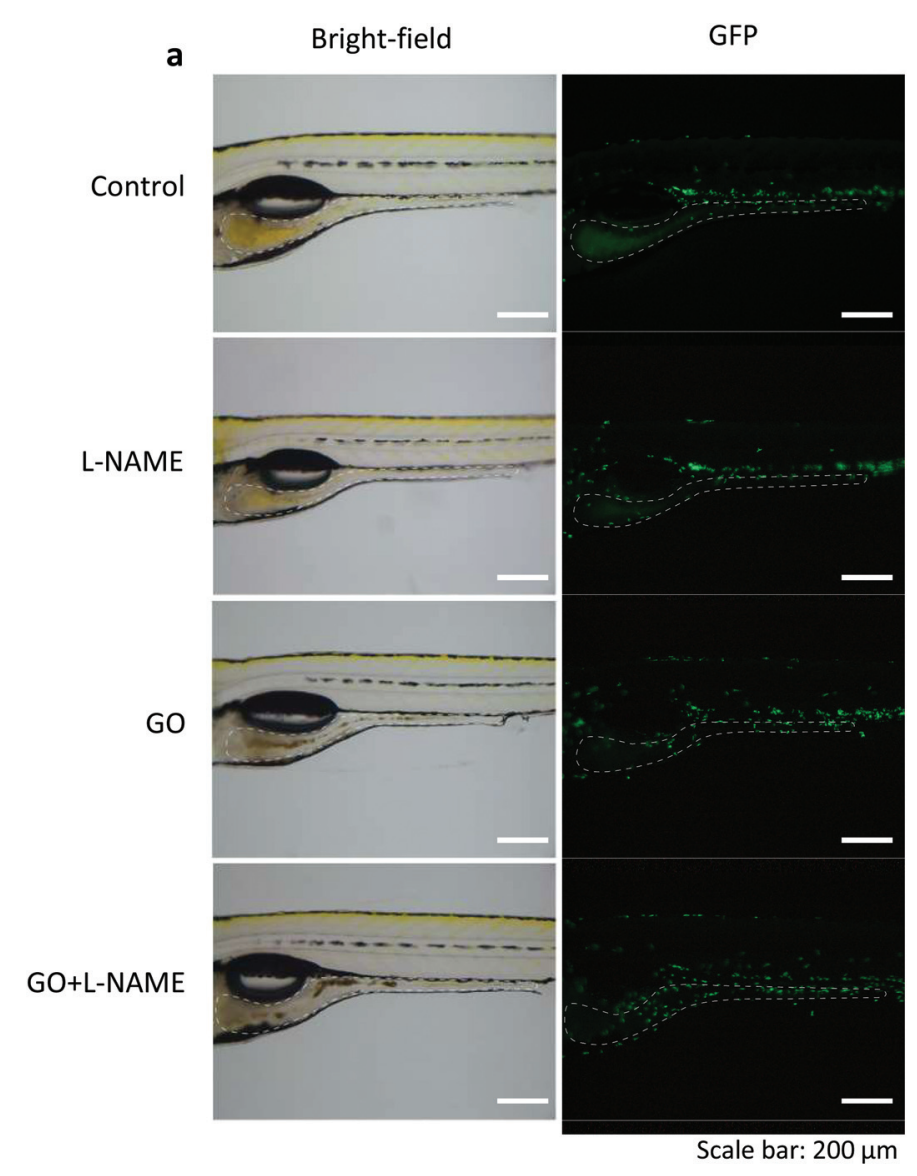

b

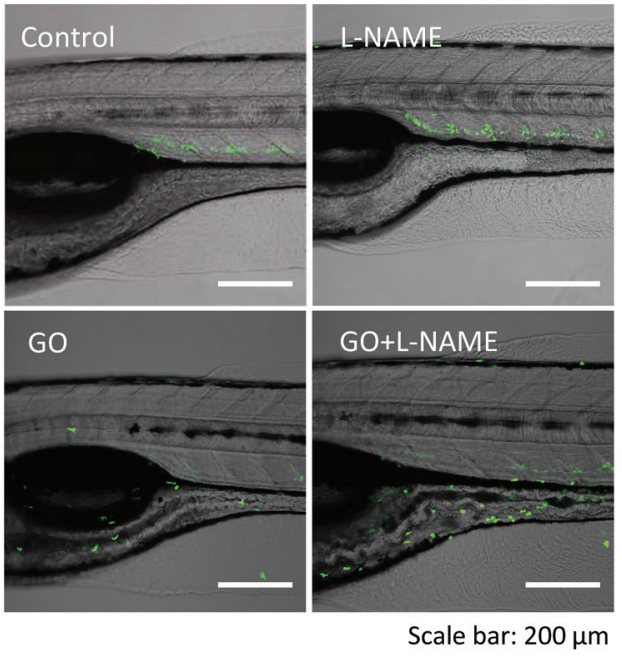

C

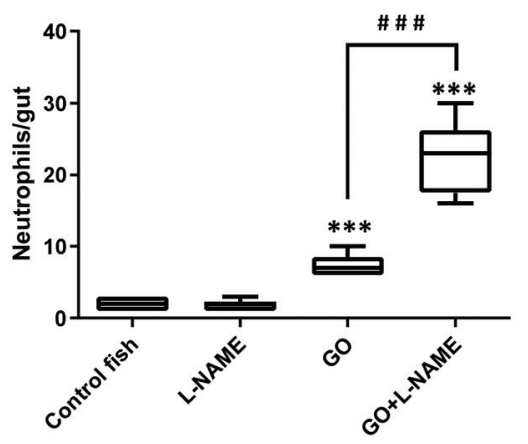

Fig. 6 Inflammation in the gastro-intestinal tract of zebrafish is enhanced when NO-dependent GO degradation is blocked. (a) The transgenic zebrafish strain $T g(m p x: e G F P)$ with GFP-labeled neutrophils was used to visualize the movement of neutrophils. Fluorescence microscopy shows that GO exposure resulted in the migration of cells to the gut, while inhibition of NOS with L-NAME led to more neutrophil infiltration. The gut is indicated by the white dashed line. (b) Confocal images of zebrafish larvae after exposure to GO \pm L-NAME. (c) Quantification of neutrophils migrated to the gut. L-NAME alone had no effect on neutrophil migration. The numbers were quantified based on 10 fish per treatment. Student's $t$-test was used to evaluate statistical significance between the treated fish and untreated control fish (* $\left.p<0.05,{ }^{* *} p<0.01,{ }^{* * *} p<0.001\right)$, as well as GO-

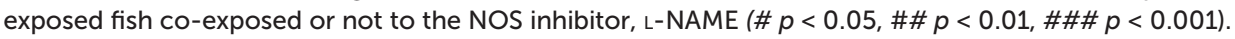


at this stage. Indeed, the overall role of NO in inflammation in mammals remains unresolved as both pro- and anti-inflammatory effects of NO and related nitrogen species have been reported. ${ }^{24,25}$ Anti-inflammatory effects of NO in mammals involve, for instance, inhibition of leucocyte activation and recruitment by endothelial cell NO. ${ }^{26}$ It should be noted that we used both pharmacological and genetic approaches to inhibit or silence iNOS in the zebrafish model. This, coupled with our in vitro results, has revealed a role for NO in the degradation of GO. However, we have not explored the different cell populations that may potentially produce NO, though iNOS is known to be expressed in many cell types in humans. We presumed that NO is generated by gastrointestinal cells in the present model and that NO (produced by iNOS) together with superoxide radicals (produced by NADPH oxidase) combined to produce the highly reactive peroxynitrite anion $\left(\mathrm{ONOO}^{-}\right)$.

\section{Conclusions}

In conclusion, we have shown that GO can be degraded in vitro and in vivo in an NO-dependent manner. This study is the first to demonstrate biodegradation of GO in zebrafish. Furthermore, while many deleterious reactions have been attributed to peroxynitrite, ${ }^{27}$ the present results suggest that this potent oxidant may also contribute to the innate defense against foreign intrusion insofar as it is shown here to participate in the degradation of GO. We also found that the degradation of GO reduces the inflammation (evidenced as neutrophil infiltration) triggered upon ingestion of GO. These findings shed new light on the biological fate of GO and further support the view that GO is a biodegradable material. ${ }^{28}$

\section{Conflicts of interest}

There are no conflicts to declare.

\section{Acknowledgements}

We thank Dr Jens Sommertune (RISE Research Institute of Sweden, Stockholm) for assistance with the confocal Raman analysis, and Dr Neus Lozano (ICN2, Barcelona) for assistance with the analysis of the material characterization data. We also thank Dr Ahmed Sultan (Karolinska Institutet, Stockholm) for generously providing the neutrophil reporter line, and the KI zebrafish core facility for assistance with fish husbandry. This work was supported by the European Commission through the Graphene Flagship (grant no. 785219).

\section{References}

1 B. Fadeel, C. Bussy, S. Merino, E. Vázquez, E. Flahaut, F. Mouchet, L. Evariste, L. Gauthier, A. J. Koivisto, U. Vogel,
C. Martín, L. G. Delogu, T. Buerki-Thurnherr, P. Wick, D. Beloin-Saint-Pierre, R. Hischier, M. Pelin, F. Candotto Carniel, M. Tretiach, F. Cesca, F. Benfenati, D. Scaini, L. Ballerini, K. Kostarelos, M. Prato and A. Bianco, ACS Nano, 2018, 12, 10582-10620.

2 G. P. Kotchey, B. L. Allen, H. Vedala, N. Yanamala, A. A. Kapralov, Y. Y. Tyurina, J. Klein-Seetharaman, V. E. Kagan and A. Star, ACS Nano, 2011, 5, 2098-2108.

3 R. Kurapati, J. Russier, M. A. Squillaci, E. Treossi, C. Ménard-Moyon, A. E. Del Rio-Castillo, E. Vazquez, P. Samorì, V. Palermo and A. Bianco, Small, 2015, 11, 39853994.

4 S. P. Mukherjee, A. R. Gliga, B. Lazzaretto, B. Brandner, M. Fielden, C. Vogt, L. Newman, A. F. Rodrigues, W. Shao, P. M. Fournier, M. S. Toprak, A. Star, K. Kostarelos, K. Bhattacharya and B. Fadeel, Nanoscale, 2018, 10, 11801188.

5 C. Martín, A. Ruiz, S. Keshavan, G. Reina, D. Murera, Y. Nishina, B. Fadeel and A. Bianco, Adv. Funct. Mater., 2019, 29, 1-11.

6 R. Kurapati, S. P. Mukherjee, C. Martín, G. Bepete, E. Vázquez, A. Pénicaud, B. Fadeel and A. Bianco, Angew. Chem., Int. Ed., 2018, 11722-11727.

7 B. L. Allen, P. D. Kichambare, P. Gou, I. I. Vlasova, A. A. Kapralov, N. Konduru, V. E. Kagan and A. Star, Nano Lett., 2008, 8, 3899-3903.

8 J. Russier, C. Ménard-Moyon, E. Venturelli, E. Gravel, G. Marcolongo, M. Meneghetti, E. Doris and A. Bianco, Nanoscale, 2011, 3, 893-896.

9 K. Bhattacharya, R. El-Sayed, F. T. Andón, S. P. Mukherjee, J. Gregory, H. Li, Y. Zhao, W. Seo, A. Fornara, B. Brandner, M. S. Toprak, K. Leifer, A. Star and B. Fadeel, Carbon, 2015, 91, 506-517.

10 F. T. Andôn, A. A. Kapralov, N. Yanamala, W. Feng, A. Baygan, B. J. Chambers, K. Hultenby, F. Ye, M. S. Toprak, B. D. Brandner, A. Fornara, J. KleinSeetharaman, G. P. Kotchey, A. Star, A. A. Shvedova, B. Fadeel and V. E. Kagan, Small, 2013, 9, 2721-2729.

11 V. E. Kagan, A. A. Kapralov, C. M. St. Croix, S. C. Watkins, E. R. Kisin, G. P. Kotchey, K. Balasubramanian, I. I. Vlasova, J. Yu, K. Kim, W. Seo, R. K. Mallampalli, A. Star and A. A. Shvedova, ACS Nano, 2014, 8, 5610-5621.

12 D. Elgrabli, W. Dachraoui, C. Ménard-Moyon, X. J. Liu, D. Bégin, S. Bégin-Colin, A. Bianco, F. Gazeau and D. Alloyeau, ACS Nano, 2015, 9, 10113-10124.

13 K. Bhattacharya, C. Sacchetti, P. M. Costa, J. Sommertune, B. D. Brandner, A. Magrini, N. Rosato, N. Bottini, M. Bottini and B. Fadeel, Adv. Healthcare Mater., 2018, 7, 1-11.

14 C. Szabó, H. Ischiropoulos and R. Radi, Nat. Rev. Drug Discovery, 2007, 6, 662-680.

15 I. S. Sohal, K. S. O'Fallon, P. Gaines, P. Demokritou and D. Bello, Part. Fibre Toxicol., 2018, 15, 29.

16 A. Pietroiusti, E. Bergamaschi, M. Campagna, L. Campagnolo, G. De Palma, S. Iavicoli, V. Leso, A. Magrini, M. Miragoli, P. Pedata, L. Palombi and I. Iavicoli, Part. Fibre Toxicol., 2017, 14, 47. 
17 J. O. Lundberg, E. Weitzberg and M. T. Gladwin, Nat. Rev. Drug Discovery, 2008, 7, 710.

18 L. I. Zon and R. T. Peterson, Nat. Rev. Drug Discovery, 2005, 4, 35-44.

19 C. M. Girish, A. Sasidharan, G. S. Gowd, S. Nair and M. Koyakutty, Adv. Healthcare Mater., 2013, 2, 1489-1500.

20 D. Guarnieri, P. Sánchez-Moreno, A. E. Del Rio Castillo, F. Bonaccorso, F. Gatto, G. Bardi, C. Martín, E. Vázquez, T. Catelani, S. Sabella and P. P. Pompa, Small, 2018, 14, 1800227.

21 V. E. Kagan, N. V. Konduru, W. Feng, B. L. Allen, J. Conroy, Y. Volkov, I. I. Vlasova, N. A. Belikova, N. Yanamala, A. Kapralov, Y. Y. Tyurina, J. Shi, E. R. Kisin, A. R. Murray, J. Franks, D. Stolz, P. Gou, J. Klein-Seetharaman, B. Fadeel, A. Star and A. A. Shvedova, Nat. Nanotechnol., 2010, 5, 354-359. 22 A. A. Shvedova, A. A. Kapralov, W. H. Feng, E. R. Kisin, A. R. Murray, R. R. Mercer, C. M. St. Croix, M. A. Lang,
S. C. Watkins, N. V. Konduru, B. L. Allen, J. Conroy, G. P. Kotchey, B. M. Mohamed, A. D. Meade, Y. Volkov, A. Star, B. Fadeel and V. E. Kagan, PLoS One, 2012, 7, e30923.

23 S. A. Renshaw, C. A. Loynes, D. M. I. Trushell, S. Elworthy, P. W. Ingham and M. K. B. Whyte, Blood, 2006, 108, 39763978.

24 J. O. Lundberg, J. M. Lundberg, K. Alving and E. Weitzberg, Nat. Med., 1997, 3, 30-31.

25 P. Kubes, Gut, 2000, 47, 6-9.

26 P. Kubes, M. Suzuki and D. N. Granger, Proc. Natl. Acad. Sci. U. S. A., 1991, 88, 4651-4655.

27 S. Pfeiffer, B. Mayer and B. Hemmens, Angew. Chem., Int. Ed., 1999, 38, 1714-1731.

28 K. Bhattacharya, S. P. Mukherjee, A. Gallud, S. C. Burkert, S. Bistarelli, S. Bellucci, M. Bottini, A. Star and B. Fadeel, Nanomedicine, 2016, 12, 333-351. 